\title{
Radiology Nursing: A Little-Known Speciality in Zambia
}

Osward Bwanga $^{1 *}$, Ernest Chanda $^{2}$, Alex Mwale $^{3}$, Biemba Kahalu Maliti $^{4}$

${ }^{T}$ Midland Regional Hospital at Tullamore, Radiology Department, Co. Offaly, Ireland

${ }^{2}$ Cancer Diseases Hospital, Radiotherapy Department, Lusaka, Zambia

${ }^{3,4}$ Cancer Diseases Hospital, Nursing Department, Lusaka, Zambia

\section{*Corresponding Author}

Osward Bwanga

\author{
Article History \\ Received: 15.07 .2020 \\ Accepted: 24.07 .2020 \\ Published: 26.07.2020
}

\begin{abstract}
Nursing is a healthcare profession which encompasses the care of patients in different settings. One of these settings is the radiology department. A radiology nurse cares for patients undergoing imaging examinations and treatment. Currently, 15 radiology nurses are working in Zambia against a population of 17 million. The radiology department is a complex environment that allows radiology nurses to work within a larger term of imaging professionals and a range of imaging equipment and patients. A radiology nurse integrates the knowledge and skills of nursing with that of imaging to ensure optimal and safe care of patients. This highlights the specialist nature of this role. However, nurses have little exposure to this speciality and nursing students are rarely attached to the radiology department during their clinical placements. This has kept radiology nursing a little-known and not an obvious specialisation option for nurses in Zambia. Therefore, this article aims to review and discuss the role of a radiology nurse in imaging examinations as well as the opportunities and challenges for developing this nursing speciality in Zambia. It is anticipated that this article will bring awareness about radiology nursing within the nursing profession and the need to develop this important speciality.
\end{abstract}

Keywords: Imaging, Radiologist, Radiology Nursing, Radiographer, Zambia.

\section{INTRODUCTION}

Zambia is a landlocked country in Southern Africa with a population of approximately 17 million. The Ministry of Health (MOH) is responsible for health management in the country. The General Nursing Council of Zambia (GNCZ) is a regulatory body for nursing professionals which was established in 1970 under the Nurses and Midwives Act No. 55 of 1970 [1]. This act was later amended in 1997 [2] to expand the scope of practice for nurses and midwives working in the country. The amended act resulted in the establishment of different nursing specialities, such as midwifery, theatre and critical care nursing which are well known. While other nursing specialities are little-known but very essential in the delivery of quality healthcare. One of such specialities is radiology nursing [3].

A radiology nurse is a specialised nursing professional who cares for patients before, during and after the imaging procedure or treatment. In Zambia, the main imaging services available include general radiography, fluoroscopy, computed tomography (CT), ultrasonography (US), mammography, magnetic resource imaging (MRI), Dual-energy X-ray absorptiometry (DEXA), nuclear medicine (NM) and catheterisation laboratory (Cath Lab). Some of the imaging services such as those involving the use of contrast media and interventional procedures are high-risk procedures were patients' acuities from stable to critically ill $[3,4]$. Thus, the services of a radiology nurse who integrates the knowledge and skills of nursing with that of imaging to ensure optimal and safe care of patients are of paramount importance in quality healthcare delivery.

In Zambia, there are currently 15 radiology nurses working in the Ministry of Health. Amongst these, three are trained in Cath lab and one in nuclear medicine. This article aims to review and discuss the role of a radiology nurse in imaging examinations which require nursing care as well as opportunities and challenges of radiology nursing in Zambia.

Copyright @ 2020: This is an open-access article distributed under the terms of the Creative Commons Attribution license which permits unrestricted use, distribution, and reproduction in any medium for non commercial use (NonCommercial, or CC-BY-NC) provided the original author and source are credited. 


\section{Imaging services at the university teaching hospital (UTH)}

In Zambia, all radiology nurses are based at the University Teaching Hospital (UTH) of Lusaka. UTH is the largest and the main referral hospital in the country with a bed capacity of 1800 [5]. The radiology department at UTH is equipped with a range of imaging modalities. However, this section focuses on imaging modalities and procedures undertaken at UTH, which require the services of a radiology nurse: fluoroscopy, CT, ultrasound, nuclear medicine (NM), Cath lab and interventional radiology. Ehrlich and Coakes [6] define interventional radiology as any procedure involving the insertion of a cannula, catheter or wire into a patient under the imaging guidance for diagnostic and/or therapeutic purposes. In most cases, a radiology nurse explains the procedure and obtain informed consent from the patient, performs intravenous (IV) cannulation, ensure the availability of emergency drugs, administer medications, monitor vital signs and help patients with their personal needs [4,8]. A detailed discussion of imaging modalities and procedures is outside the scope of this article.

General X-ray equipment - it is used to perform general radiography examinations of amongst others, the chest, skull, spine, extremities, pelvis and abdomen. It is also used to perform specialised radiological procedures such as intravenous urogram (IVU) which may require the services of a radiology nurse. An IVU is a functional study of the urinary system accomplished by the intravenous injection of contrast media [6]. Figure 1 shows the general X-ray equipment.

Fluoroscopy - is continuous X-ray imaging, which allows dynamic real-time visualisation of the imaged structures [4]. The most common fluoroscopy procedures performed where the service of a radiology nurse is required include a barium enema, angiogram, hysterosalpingography (HSG), urethrogram, sialogram and fistulogram. Figure 2 shows a fluoroscopy X-ray unit.

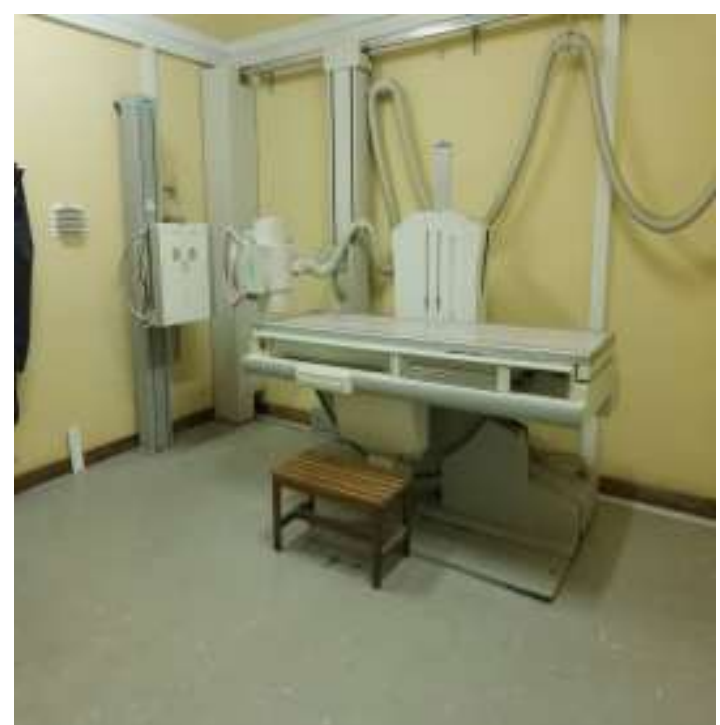

Fig-1: One of the general X-ray units at UTH

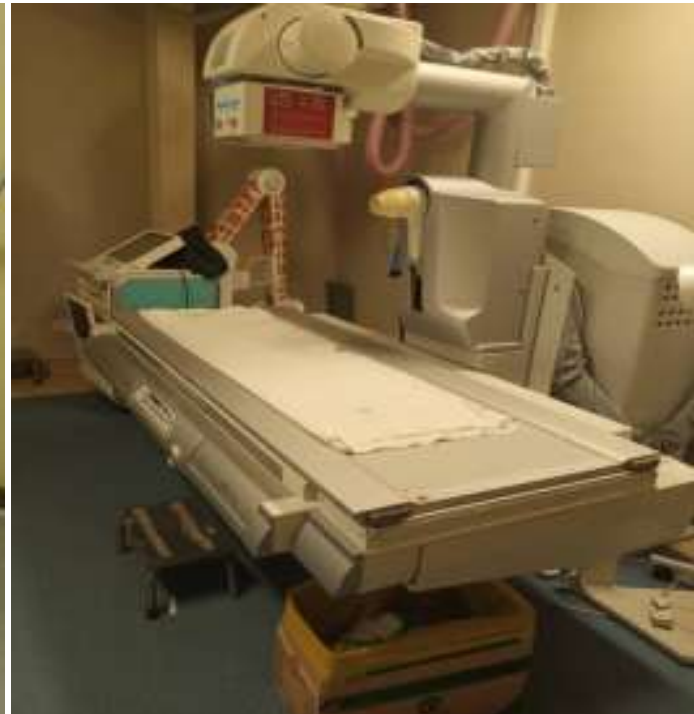

Fig-2: Fluoroscopy unit at $\mathrm{CDH}$

Ultrasonography (US) - is an imaging technique which uses high-frequency sound waves to produce images [4]. Ultrasound does not use ionising radiation. Percutaneous drainages procedures, steroid injections and biopsies are the common ultrasound-guided procedures undertaken which require nursing care. Figure 3 shows an ultrasound unit.

Computed tomography (CT) - is an imaging technique which uses X-rays to produce cross-sectional images of the human body [6]. CT produces more detailed information than conventional (plain film) radiography. The most common CT guided procedures which require nursing care include drainage procedures, angiograms, biopsies and steroid injections. In CT, nursing care is required for the intravenous (IV) cannulation and contrast administration before imaging as well as monitoring patients for adverse contrast reactions [4,6]. Figure 4 shows a CT scanner. 


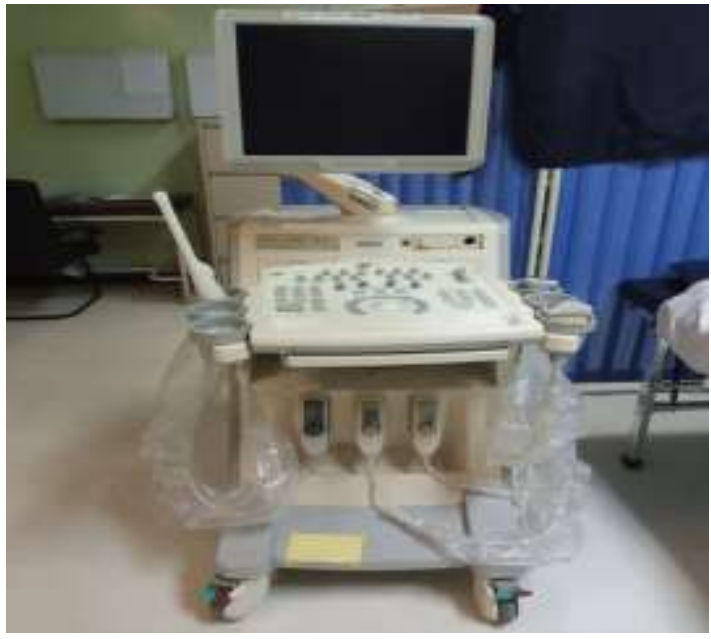

Fig-3: One of the ultrasound units at UTH

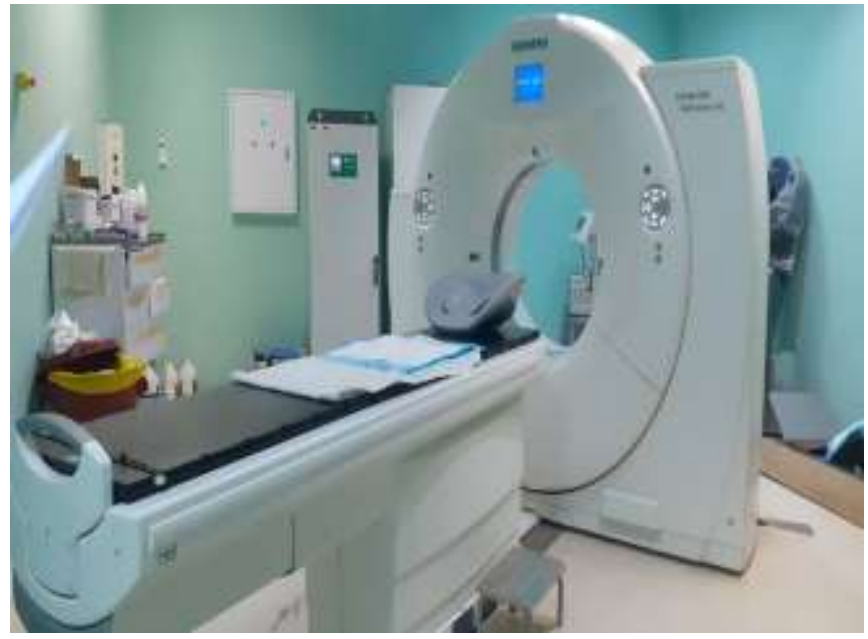

Fig-4: Computed tomography unit at $\mathrm{CDH}$

Nuclear medicine (NM) - is an imaging modality that uses a small amount of radioactive material or radiopharmaceuticals to provide information about organs and tissues [6]. Specific duties of radiology nurses working in nuclear medicine include stress testing patients for myocardial perfusion imaging and administration of radioisotopes. Figure 5 shows a nuclear medicine unit.

Catheterisation laboratory (Cath lab) - is both an imaging examination and treatment room in the radiology department where procedures are carried out for diagnostic and interventional purposes [7]. It is called Cath lab because catheters are inserted into a vein or artery and directed to the area of interest under the fluoroscopic imaging guidance [8]. The Cath lab is staffed by a team of different healthcare professionals such as radiologists, cardiologists, radiographers, and radiology nurses (Cath lab nurses). The procedures performed in the Cath lab mainly involve the examination and treatment of heart conditions. The common diagnostic procedures performed at UTH are angiograms, while treatment procedures include repairs of septal defects, stenting of coronary vessels, placement of pacemakers and fibroid embolization. All procedures performed in the Cath lab requir-e the services of a radiology nurse. Figure 6 shows a Cath lab suite.

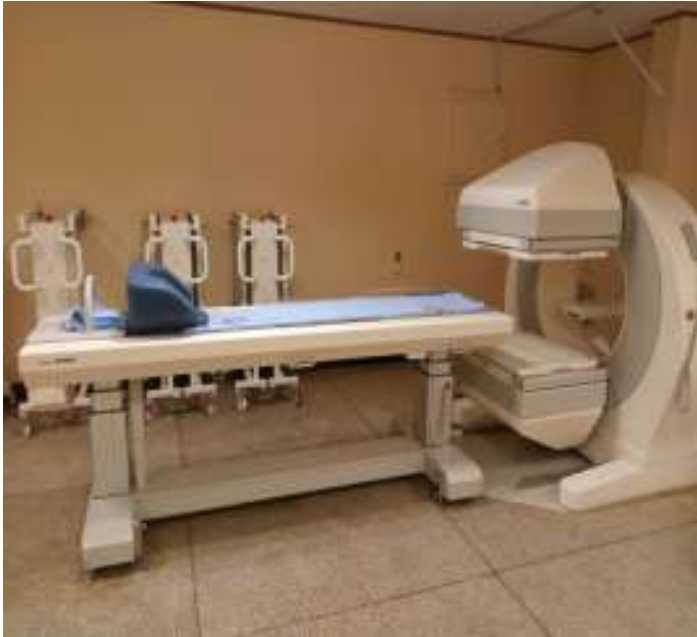

Fig-5: Nuclear medicine unit at UTH

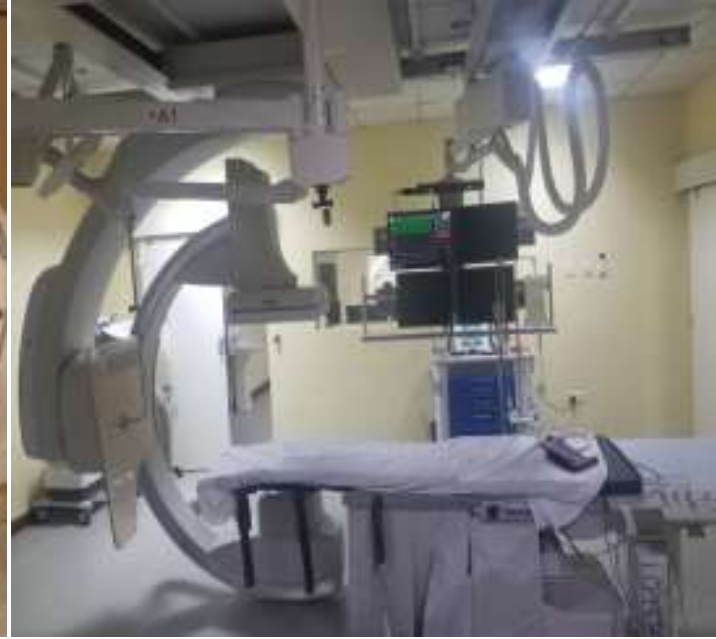

Fig-6: Cath lab unit at UTH

\section{Roles of a radiology nurse in imaging}

In Zambia to become a radiology nurse, someone must be trained and registered as a registered nurse with the General Nursing Council of Zambia (GNCZ). The radiology department is a complex clinical environment that allows radiology nurses to work within a larger team of imaging professionals, such as radiologists and radiographers as well as with a range of imaging equipment and patients. This makes the role a complex and demanding one. Patient care for most of the examinations and procedures or treatment is provided by radiographers. However, for complex procedures which require sedation or scrub assistance, patient care must be provided by a radiology nurse [3, 4, 8]. In the radiology department, the roles of a radiology nurse can be grouped into three: pre-procedural nursing care, nursing care during the procedure and post-procedural nursing care $[3,4,9]$. 
Pre-procedural nursing care - this role encompasses all nursing care given to the patient before the imaging procedure and treatment. The role involves patient preparation and assessment of the patient's suitability for the radiological examination or procedure [4]. Each imaging procedure and treatment requires different and specific patient preparation and assessment [3]. The pre-procedural nursing care starts with the establishment of a good relationship with the patient and family members or carers [6]. The radiology nurse also checks the pregnancy status of women of childbearing age for any radiological procedure involving the pelvis. For procedures involving intravenous (IV) injection of contrast media or administration of any drug, the radiology nurse also performs IV cannulation. Other common preprocedural tasks undertaken by a radiology nurse include the following $[3,8,9]$ :

- Explaining the procedure to the patients and obtaining informed consent.

- Check the patient's known allergies before administering contrast media.

- Check the patient's renal function and blood for urea and electrolytes (U\&E) which is vital to know before the administration of contrast media.

- $\quad$ Prepare nursing equipment for the safe care of the patient.

- Identify potential complications of various drugs during the procedure.

- Communicate any concerns with a radiologist and radiographer.

- Daily checking of the crash trolley and to ensure that it is accessible in case of any need during a procedure.

- Document all necessary information in the patient's folder or electronically.

To effectively perform this pre-procedural role, the nurse needs to possess knowledge of all relevant imaging examinations and procedures to be able to inform the patient and answer any questions the patient may have [8,9]. However, some of the patient's questions can only be answered by the radiographer and/or radiologist.

Nursing care during the procedure - Most of the imaging examinations and procedures do not require the services of a radiology nurse. Patient care is carried out by radiographers, radiologists or supporting staff. However, complex procedures, such as interventional radiology require two nurses: a radiology nurse and a registered nurse [6,8]. The radiology nurse working directly with the radiologist within the sterile field during the procedure and act as a scrub nurse, while the registered nurse monitors the patient and acts as a circulating nurse [6]. According to the Royal College of Nursing and Royal College of Radiologists [8], the duties of a radiology nurse (scrub nurse) include the following:

- $\quad$ Preparing of sterile instruments and equipment ready for the procedure.

- Assembling sutures, needles, blades and other necessary sterile equipment with the assistance of the circulating nurse or radiographer.

- Dispensing catheters, swabs, and other equipment as needed; cleaning and replacing catheters and wires as required.

- Anticipating the needs of the clinical radiologist.

- Disposing of all needles, blades and other contaminated equipment in accordance with the hospital policy.

To effectively perform the nursing care during the procedure, a radiology nurse needs an in-depth knowledge of each procedure so that he or she can anticipate the needs of the clinical radiologist, and to ensure that the circulating nurse or radiographer is aware of the needs to collect specialist items as required $[3,6,9]$.The radiology nurse should also understand and apply the three control measures of external radiation: shielding, distance and time. The use of shielding (lead apron or mobile lead screen), a greater distance from the radiation source, and reducing the time of exposure to radiation minimises exposure to external radiation [10].

Post-procedural nursing care - In the radiology department most of the patient care after the diagnostic imaging is undertaken by radiographers. However, the radiology nurse provides post-procedural nursing care to complex procedures such as interventional radiology. For imaging examinations involving IV administration of contrast media, patients are generally asked to wait for observation due to delayed reactions of contrast media [4, 6]. The early recognition of the adverse reaction of contrast media is part of the remit of the radiology nurse [8]. Following some imaging procedures and treatment, patients may require intensive nursing care in the recovery room before being discharged or transferred back to the ward [3,6,9]. According to the Royal College of Nursing and Royal College of Radiologists [8], the following are some of the post-procedural nursing care undertaken by the radiology nurse:

- Providing patient comfort as most often the post-procedural patient is restless and can easily come to lie in an unsatisfactory position, which can cause undue pain and cause pressure necrosis.

- Recording the early signs of complications and acting upon these as appropriate. These include maintenance of a patient's airway and observation of the rate and depth of respiration.

- Maintenance of an adequate circulation as many patients become hypotensive during and after interventional procedures due to vagal stimulation or unforeseen bleeding.

- Observation of puncture site and relevant pulses, such as foot and radial.

- Neurological observations following cerebrovascular interventions, vertebroplasty and aortic stent grafting. 
All the above stated post-procedural nursing care duties relates to nursing and cannot be undertaken by radiographers. It should be noted that radiographers' training is intrinsically different from that of nurses. Therefore, these stated nursing tasks should be undertaken by a radiology nurse.

\section{Strengths and opportunities for developing radiology nursing in zambia}

There are some strengths and opportunities for developing radiology nursing in Zambia. The main strengths and opportunities are presented in Table 1.

Table-1: Strengths and opportunities for developing radiology nursing in Zambia

\begin{tabular}{|l|l|}
\hline No & \multicolumn{1}{c|}{ Strengths and opportunities } \\
\hline 1 & There has been an increase in demand for diagnostic imaging and radiology nursing services \\
\hline 2 & Ministry of Health and General Nursing Council (GNC) are promoting specialisation in nursing \\
\hline 3 & $\begin{array}{l}\text { There are readily available, educational infrastructure and teaching staff for developing an educational training } \\
\text { programme in radiology nursing }\end{array}$ \\
\hline
\end{tabular}

Firstly, different modern imaging modalities have been introduced into the Zambian healthcare system in the last two decades. Currently, there are $17 \mathrm{CT}$ scanners, 16 mammographic units, 5 MRI scanners, 1 nuclear medicine scanner, 2 dual-energy X-ray absorptiometry (DEXA) unit and 1 Cath lab in the country. There is a reason to assume that more diagnostic imaging modalities will be purchased and installed in both public and private health facilities countrywide, including new services such as Positron Emission Tomography-Computed Tomography (PET/CT). The Ministry of Health has recently introduced a radiology specialist training programme for medical doctors. As the number of imaging services and radiologists increases in the country, so does the demand for radiology nurses. This also means readily available jobs in this specialised field of nursing.

Secondly, the General Nursing Council of Zambia (GNCZ) and Ministry of Health are promoting specialisation in nursing. Since the establishment of the Nurses and Midwives Act No. 31 of 1997 [2] which aimed at expanding the scope of practice of nursing professionals, several accredited training programmes have been developed to equip nurses and midwives with specialised knowledge and skills in different areas. According to the General Nursing Council of Zambia [11], there are eight accredited specialised postgraduate educational programmes including midwifery, critical care nursing, operating theatre nursing, psychiatric nursing, paediatric nursing, public health nursing, ophthalmic nursing, and clinical teaching. The Ministry of Health also established Levy Mwanawasa Medical University (LMMU) in 2018 for training health professionals. The LMMU is a 3,000 student capacity campus, including a 1,000 capacity National Health Reference Library, and 200 bed National Skills Laboratory Complex [12]. The interest and support from key stakeholders make it possible to develop radiology nursing training programme.

Thirdly, there is readily available educational infrastructure and teaching staff to support the development of a radiology nursing training programme in Zambia. The main five public nursing training institutions at LMMU, University of Zambia (UNZA) School of Nursing, Lusaka Schools of Nursing and Midwifery, Ndola Schools of Nursing and Midwifery, and Kitwe Schools of Nursing and Midwifery have the infrastructure to support the radiology nursing training programme. There are also radiology departments offering a variety of diagnostic imaging services and radiologists in the stated training centres/teaching hospitals which can be used in clinical practice. The already available radiology nurses, radiologists, radiographers and consultant doctors could teach the trainees. There is also the availability of Health Professions Education Programmes at LMMU and UNZA to support the teaching staff in acquiring knowledge and skills in lecturing. The available resources make it possible to develop a training programme in radiology nursing in Zambia.

\section{Education and training in radiology nursing}

Due to greater advancement in imaging technology and procedures, a radiology nurse must have a high level of knowledge and skills [3]. The Royal College of Nursing and Royal College of Radiologists [8], adds that the highly technical field of radiology nursing requires knowledge, skills, creativity and versatility that should be offered by competent individuals, suitably trained and qualified to perform the required tasks to the highest standards. This requires undertaking a specialised postgraduate training by any registered nurse who wants to take up the role of caring for patients in the radiology department. Unfortunately, there is no training programme in radiology nursing in Zambia. Most of the nurses working in radiology departments in Zambia have only undertaken work-based learning (on-job training), except for one who has done formal training in nuclear medicine. However, work-based learning may be opportunistic and based solely on the available examinations and procedures [13]. Besides, there is a lack of theory in work-based learning which is vital in the learning process as it guides learners in turning the knowledge into clinical practice. 
Given the above, there is a need to develop a postgraduate training programme in radiology nursing locally. The focus of education and training is to impart knowledge to nurses working in radiology departments about different imaging procedures and how to apply their nursing skills to patients in this specialised area. The curriculum may include a basic understanding of different imaging modalities, common drugs used in radiology, contrast media and life support, vascular radiology, hepatobiliary radiology, gastro-intestinal radiology, urological radiology, reproductive system radiology and interventional radiology, as well as radiation protection [6,8]. Radiology nurses should be knowledgeable about measures to protect patients, themselves, other clinical staff and members of the public from radiation, which is a statutory requirement under the ionising radiation protection act of 2011 of the Republic of Zambia [14]. The nursing education providers should work with the GNCZ in developing and accrediting the training programme.

\section{Weaknesses and threats to the development of radiology nursing in Zambia}

There are some weaknesses and threats to the development of radiology nursing in Zambia. The main weaknesses and threats are summarised in Table 2.

Table-2: Weaknesses and threats to the development of radiology nursing in Zambia

\begin{tabular}{|l|l|}
\hline No & \multicolumn{1}{c|}{ Weaknesses and threats } \\
\hline 1 & Lack of awareness about radiology nursing \\
\hline 2 & Increase in workload due to shortage of radiology nurses \\
\hline 3 & $\begin{array}{l}\text { Non-availability of a local training programme, continuing professional development (CPD) learning } \\
\text { activities and educational sponsorship in radiology nursing }\end{array}$ \\
\hline
\end{tabular}

Firstly, there is a lack of awareness of radiology nursing in Zambia. Generally, nurses working outside the radiology department have little exposure to this speciality. Also, there is no radiology in the undergraduate nursing curriculums and nursing students are rarely exposed to the radiology department for clinical practice. This situation has kept radiology nursing a little-known and underdeveloped speciality and not an obvious option for registered nurses considering postgraduate studies. There is a need for radiology nurses, radiographers and radiologists to bring awareness about this important speciality in the delivery of quality radiology services.

Secondly, there is an increase in workload due to shortage of radiology nurses to work in all teaching hospitals in the country with radiologist's services. The increase in workload has resulted in the use of registered nurses who have no specialised training to offer nursing care to patients undergoing imaging procedures. Registered nurses may lack knowledge and skills of imaging examinations and procedures as well as radiation protection which is vital for the optimal and safe care of patients in this area of medicine $[6,8]$. Therefore, there is a need to increase the number of radiology nurses to meet the demand.

Thirdly, there is no local postgraduate radiology nursing training programme to equip nurses working in this area with specialised knowledge and skills of imaging. To obtain a qualification in radiology nursing, one has to be enrolled at a training institution abroad. However, enrolling outside Zambia also is a challenge due to a lack of government sponsorship. There is also a lack of continuing professional development (CPD) learning activities in this speciality. CPD can help radiology nurses to maintain competence and keep up to date with the current development in this nursing speciality [8]. There is a necessity to integrate this subject in the nursing curriculum and develop a radiology nursing training programme.

\section{Conclusion}

This article reveals that radiology nursing is a little-known and underdeveloped nursing speciality in Zambia. The increasing demand for imaging services and the number of radiologists in the country requires the corresponding increase in the number of radiology nurses. Therefore, there is a need to bring awareness of this field within the nursing profession and establish a training programme. It is highly recommended that all nursing students should have some exposure to radiology nursing during clinical placements. Radiologists, radiographers and radiography technologists should also make presentations to the nurses and nursing students about this speciality during their annual conferences. These strategies will raise awareness and understanding of radiology nursing which is vital in the management of patients undergoing imaging examinations and procedures.

\section{ACKNOWLEGEMENT}

Our acknowledgement first goes to our late colleague Kaela Mooya, former radiographer at UTH, radiology department who contributed greatly to this article. Secondly, to Sr Esnart Lungu Chanda, the radiology nurse at UTH for her insight and moral support. The authors would also like to express appreciation to the University Teaching Hospital (UTH) and Cancer Diseases Hospital (CDH), through the radiology and radiotherapy departments for the pictures of different imaging modalities included in this article. 


\section{REFERENCES}

1. Republic of Zambia. (1970). Nurses and midwives act No. 55 of 1970. Lusaka: Government Printers.

2. Republic of Zambia. (1997). Nurses and midwives act No. 31 of 1997. Lusaka: Government Printers.

3. Moyo, M. (2019). Radiology nursing: A growing speciality. American Nurse Today, 14(9): 73-75.

4. Jones, S., \& Taylor, E.J. (2006). Imaging for nurses. Oxford: Blackwell Publishing Ltd.

5. Kayembe, R.K., \& Bwanga, O. (2020). A study to explore the knowledge, attitudes and practices of student radiographers regarding feedback on clinical performance in Zambia. The South African Radiographer, 58(1): 8-14.

6. Ehrlich, R.A., \& Coakes, D.M. (2020). Patient care in radiography: with an introduction to medical imaging. 10th ed. London: Elsevier.

7. Partridge, J. (2005). Radiation in the cardiac catheter laboratory. Heart (British Cardiac Society), 91(12), 1615-1620.

8. Royal College of Nursing and Royal College of Radiologists. (2014). Guidelines for nursing care in interventional radiology. $2^{\text {nd }}$ Ed. London: RCN/RCR.

9. Blevins, S. J. (1994). The role of the radiology nurse. Radiology Management, 16 (4): 46- 48.

10. Martin, A., Harbison, S., Beach, K., \& Cole, P. (2019). An introduction to radiation protection. $7^{\text {th }}$ Ed. London: CRC Press.

11. General Nursing Council of Zambia. (2018). Approved and registered nursing and midwifery schools, colleges and universities. Lusaka: GNCZ.

12. Levy Mwanawasa Medical University. (2020). Background of Levy Mwanawasa Medical University. From http://www.cchs.ac.zm/background-levy-mwanawasa-medical-university (Accessed 4 February 2020).

13. Harden, R.M., \& Laidlaw, J. M. (2017). Essential skills for a medical teacher-An introduction to teaching and learning in medicine. $2^{\text {nd }}$ Ed. London: Elsevier.

14. Republic of Zambia. (2011). Ionising radiation protection act of 2011 (Amended). Lusaka: Government Printers. 